\title{
Ploidy studies in adenomatous polyps of the colon
}

\author{
F WHITEHEAD, LR JAR.VIS, JM SKINNER, R WHITEHEAD \\ From the Department of Pathology, Flinders Medical Centre, Bedford Park, South Australia
}

SUMMARY The nuclei of colonic adenomatous polyps and some colonic carcinomas have a normal diploid profile. The remaining carcinomas are aneuploid, and this change most probably occurs after the dysplasia that determines invasiveness, because even adenomatous polyps with carcinoma in situ are diploid.

Most adenocarcinomas of the colon originate in adenomatous polyps. Integral to the malignant process, in both adenomatous polyps and carcinomas, is a disorder of cell kinetics that manifests itself at light microscopy by variation in nuclear size, shape, and optical density, together with changed cytoplasmic appearances. These so called dysplastic changes reflect important changes in the replication, differentiation, and function of the cell. It has been shown, for example, that several malignancies may have a grossly abnormal DNA complement-that is, they are aneuploid. This is true for some but not all adenocarcinomas of the colon.' In view of the known sequence of adenomatous polyp to cancer in the colon we carried out a study of nuclear ploidy distribution in polyps to ascertain whether ploidy abnormalities similar to those in some colonic cancers are present at the adenomatous polyp stage of the disorder.

\section{Material and methods}

Paraffin embedded tissue from 16 adenomatous polyps was studied. The degree of dysplasia was assessed according to the criteria of Konishi and Morson. ${ }^{2}$ For comparison 41 cases of adenocarcinoma were also examined and staged $\mathrm{A}, \mathrm{B}$, and $\mathrm{C}$ according to the criteria of Dukes. ${ }^{3}$ Lymphocytes from reactive but otherwise normal human lymph nodes and normal human colonic epithelial cells in resected bowel mucosa from 12 cases of diverticular disease were studied as controls.

Cell suspensions were prepared according to the method of Hedley et al. ${ }^{4}$ Sections $30 \mu \mathrm{m}$ thick were cut and the wax removed with xylol. The tissue was then mechanically and enzymatically disaggregated. The cell suspensions were centrifuged on to a micro-

Accepted for publication 6 August 1985 scope slide using a Cytospin microcentrifuge and were stained using the Feulgen method. ${ }^{56}$

\section{MEASUREMENT OF INTEGRATED OPTICAL DENSITY}

Integrated optical density was measured using a microcomputer image analysis system designed in our laboratory. The hardware of the system comprised a solid state video camera (Hamamatsu C1700, Hamamatsu Photonics KK, Hamamatsu City, Japan) using a charge coupled device imaging array of $320 \times 244$ elements, a video digitiser (Micro-Works DS80 Digisector, MICRO-WORKS, Del Mar, California), a random access device with a maximum of $256 \times 256$ resolution and six bit grey values, and a microcomputer (Zenith Z100, Zenith Data Systems, Michigan). The camera was mounted on an Olympus BHS-112 (Olympus Optical Co, Tokyo, Japan) with trinocular head and C-mount adapter for the camera. All measurements were made with an S-Plan Achromat objective of $\times 40$ magnification and using a $546 \mathrm{~nm}$ interference filter with a band width of $60 \mathrm{~nm}$.

The video image was processed by a random access video digitising board that transferred a picture field of $192 \times 189$ pixels to the computer memory. Transfer of the image to display by way of a translation table gave the optimum grey scale and pseudocolour display. The features to be measured were extracted by thresholding and the creation of a binary image displayed as green on a black background. Features were edited by erasure in red or by drawing in green. The binary image was effectively used as a mask over the grey level image for automatically distinguishing features to be measured. The number of pixels was the feature area and the sum of the pixel values the integrated optical density. Sums of squares and cubes were also accumulated to calculate the coefficient of variation, skewness, and kurtosis of the feature grey level dis- 


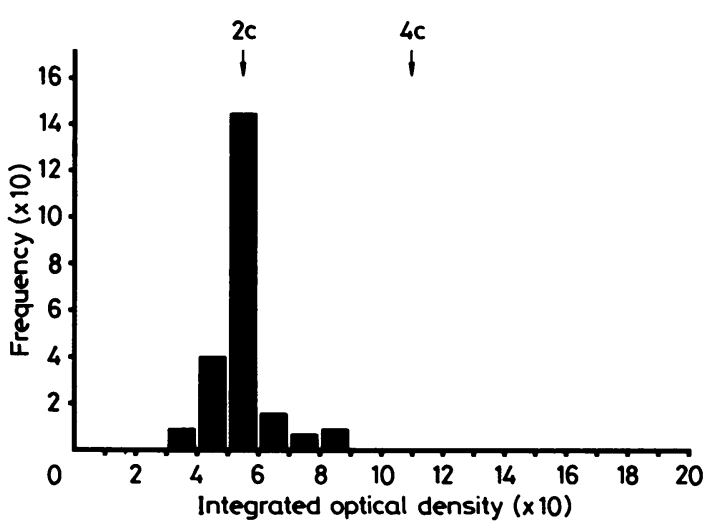

Fig. 1 DNA histogram of type 1, showing single 2c peak. This represents one type of normal profile.

tribution. The perimeter was calculated by totalling edge transitions with a diagonal correction for corner pixels.

Calibration procedures ensured that the pixel values were linearly proportional to the intensity of illumination. Correction for uneven illumination was performed by storing an image of the background. The pixel values from this store were retrieved and used to correct each corresponding pixel for every subsequent scan.

For each session of measurement the level of microscope glare was estimated. The apparent minimum transmittance of carbon particles larger than $5 \mu \mathrm{m}$ in diameter was measured and used for subsequent corrections for glare.?

The software was developed in our laboratory and permitted measurement of the integrated optical density of images of nuclei stained by Feulgen's method which was proportional to the nuclear DNA content. The measurements were made in arbitrary units. To overcome problems of variation in density of staining these units were related to measurements of a standard slide of normal human lymphocytes and colonic epithelial cells stained in each batch of specimens. A histogram of the distribution of nuclear integrated optical density was constructed for each specimen.

The overall performance was evaluated using a biological standard of rat liver nuclei. Measurements of the integrated optical density of these nuclei should be proportional to the DNA content, and the distribution should display peaks at doubled intervals. ${ }^{8}$ This test of proportionality was repeated regularly on fresh rat liver preparations for quality control and the staining techniques, the measurement system, and the operator. The calculation of ploidy by analysis of the histogram can therefore be

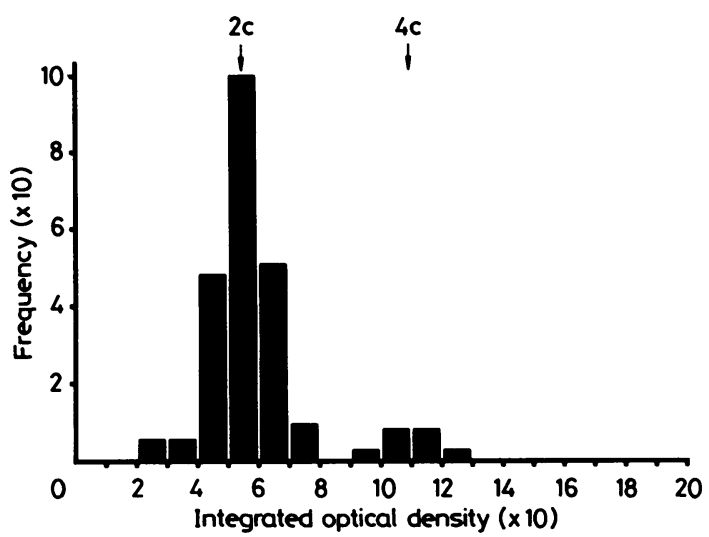

Fig. 2 DNA histogram of type 2, showing another normal distribution pattern with peaks at $2 c$ and $4 c$ and some cells in $S$ phase.

accepted as reliable for the material studied. In practice, when care was taken over the staining method and constancy of microscope adjustments, the integrated optical density of a normal lymphocyte remained within narrow limits and faulty staining could be quickly recognised by a few measurements of the control preparation. The results of ploidy analysis by this system were tested against the fluorescence activated cell sorter, using the same preparations of nuclei. Results given by both methods were identical.

\section{Results}

The results of the analysis produced four basic types of histogram (Figs. 1-4). The first type (15 cases) (Fig. 1) represented a diploid cell population in which all the cells measured were in $G_{0}$ and $G_{1}$. The

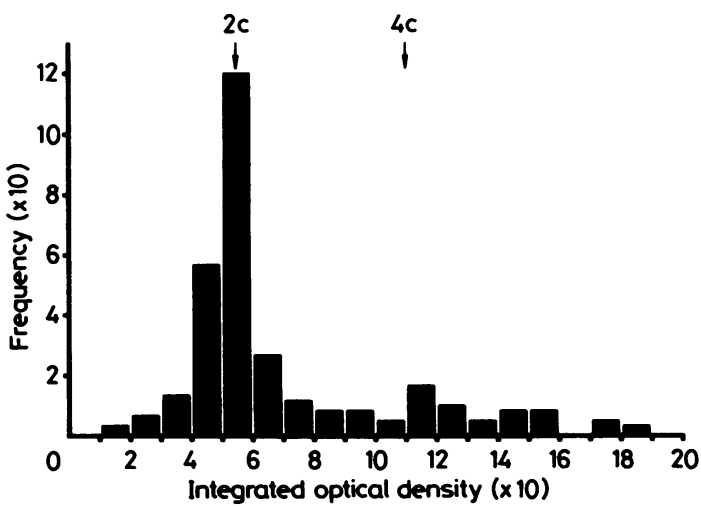

Fig. 3 Abnormal distribution of DNA in histogram of type 3. This "hyperdiploid" profile shows small DNA peaks in excess of $4 c$ peak. 


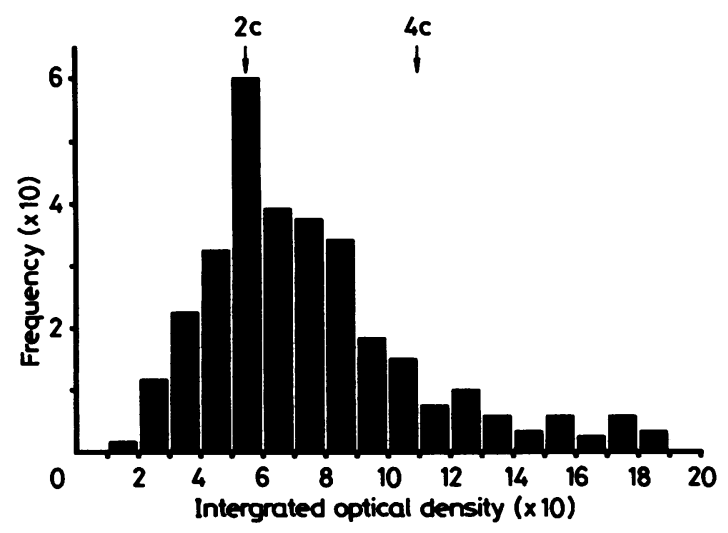

Fig. 4 Abnormal DNA profile of type 4, showing obvious aneuploid population forming a "shoulder" to right of $2 c$ peak and peaks in excess of $4 c$.

second type (10 cases) (Fig. 2) showed a large 2c peak and a smaller population of $4 \mathrm{c}$ nuclei, which were about to enter mitosis. This pattern implied a larger proliferative compartment than is depicted in Fig. 1. Both these types were normal. The third DNA profile (13 cases) (Fig. 3 ) could be described as showing a hyperdiploid distribution. It consisted of a large peak in the $2 \mathrm{c}$ region with a greater proportion of cells actively synthesising DNA, indicated by a larger number of nuclei in the $4 c$ region and in the $S$ phase region between these peaks. There were also nuclei with a hyperdiploid DNA constitutionthat is, their DNA content was beyond the normal multiple of the diploid DNA content (4c). These hyperdiploid DNA values were perhaps caused by the defective division of the nucleus during mitosis producing an unequal share of DNA between the two daughter cells." The fourth type of histogram (three cases) (Fig. 4) depicted an aneuploid DNA profile with an obvious shoulder adjacent to the 2c peak, suggesting two or more populations of cells within the same tumour. Both these last two types were abnormal. Tables 1 and 2 summarise these results.

Table 1 Adenocarcinomas

\begin{tabular}{|c|c|c|}
\hline Stage (Dukes' grading) & Histogram type & No of cases \\
\hline & $\int_{2}^{1}$ & \\
\hline A & 2 & 3 \\
\hline & 4 & 0 \\
\hline B & $\begin{array}{l}1 \\
2 \\
2\end{array}$ & $\begin{array}{l}1 \\
5\end{array}$ \\
\hline & 4 & $\begin{array}{l}2 \\
2\end{array}$ \\
\hline C & $\begin{array}{l}1 \\
2\end{array}$ & $\begin{array}{r}11 \\
2\end{array}$ \\
\hline & $\begin{array}{r}3 \\
-4\end{array}$ & $\begin{array}{l}4 \\
1\end{array}$ \\
\hline
\end{tabular}

Table 2 Adenomatous polyps

\begin{tabular}{lllll}
\hline Degree of dysplasia & \multicolumn{4}{c}{ No of cases showing different histcgram types } \\
\cline { 2 - 5 } & Type 1 & Type 2 & Type 3 & Type 4 \\
\hline Mild & 5 & 1 & 0 & 0 \\
Moderate & 4 & 2 & 0 & 0 \\
Severe & 4 & 0 & 0 & 0 \\
\hline
\end{tabular}

\section{Discussion}

This study agreed with previous findings ${ }^{1}$ in that a proportion of adenocarcinomas have a diploid DNA profile, the remainder being aneuploid. It is not known whether eventually all the adenocarcinomas would become aneuploid. As all the adenomatous polyps examined in this study were diploid (even those with severe dysplasia similar in appearance to carcinoma but without invasion ${ }^{2}$ ) and 25 of the 41 carcinomas examined were diploid, the change from diploid to aneuploid may have occurred after the event that determines the property of invasion. Furthermore, the fact that ploidy abnormality was not found in 19 adenomatous polyps and in 25 carcinomas also means that aneuploidy does not correlate well with the histological variables of dysplasia or dedifferentiation, the common histological features of malignancy. In particular, if, as suggested by Konishi and Morson, the degree of dysplasia in a polyp may determine subsequent development of further polyps or carcinoma, or both, in the same patient ${ }^{2}$ it would seem that this is not reflected by changes in ploidy distribution.

As the risk of a cancer developing in an adenomatous polyp is small ${ }^{10}$ it could be argued that the sample we examined was not large enough to detect potential malignancy. Four of the 19 adenomatous polyps contained foci of severe dysplasia, however, which, by definition, is a histological abnormality with the appearances of carcinoma but found only in epithelium that has not been invaded. Indeed, Kalus is one among many who use the term carcinoma in situ for this lesion." Nevertheless, in view of the proportions of diploid to aneuploid invasive carcinomas we must consider the possibility that examination of further adenomatous polyps would show one or more of aneuploid composition. Analysis of our data using the $\chi^{2}$ test $\left(\chi^{2}=2 \cdot 422\right.$, df $=1,0.75<p<0.90$ ) shows that if we conclude that for polyps with carcinoma in situ aneuploidy does not occur we could be confident of being correct in $75-90 \%$ of cases.

Other workers showed that ploidy abnormality in adenocarcinoma of the colon correlates well with a poor prognosis. ${ }^{1}$ In this study the confirmation of diploid distributions in adenomatous polyps and 
some adenocarcinomas suggests that ploidy abnormality takes place some time after invasion has occurred and represents a new adverse trend in a particular tumour. This does not exclude the possibility that the occurrence of aneuploidy is simply a function of time, or that all carcinomas would become aneuploid should the patient survive long enough, but it is interesting to note that Dukes's A tumours which have invaded no further than the bowel wall, if they are aneuploid, exhibit the less severe form (type 3); this re-emphasises the importance of early recognition and removal of both adenomatous polyps and carcinomas of the colon.

The findings in this study also support the view that the malignant process in cancer of the colon is a stepwise process and that with each step abnormality of the cell, either morphologically or functionally, is increased as is the likelihood of aggressive behaviour. There is clearly a case for ploidy studies on all adenocarcinomas of the colon, especially with the technique used in this study, which is simple and cost effective and makes the likelihood of the analysis becoming a routine procedure possible. As new therapeutic possibilities are introduced, early and aggressive treatment could well be implemented on the basis of such an analysis.

This work was generously supported by a grant from the Anti-Cancer Foundation of South Australia.

\section{References}

' Wolley RC, Schreiber K, Koss LG, Karas M, Sherman A. DNA distribution in human colon carcinomas and its relationship to clinical behaviour. Journal of the National Cancer Institute 1982;69:15-22.

${ }^{2}$ Konishi F, Morson BC. Pathology of colorectal adenomas: a colonoscopic survey. J Clin Pathol 1982;35:830-41.

${ }^{3}$ Dukes CE. Cancer of the rectum: an analysis of 1000 cases. Journal of Pathology and Bacteriology 1940;50:527-39.

${ }^{4}$ Hedley DW, Friedlander ML, Taylor IW, Rugg GC, Musgrove EA. Method for analysis of cellular DNA content of paraffin embedded pathological material using flow cytometry. $J$ Histochem Cytochem 1983;31:1333-5.

${ }^{5}$ Fand SB: Enrivonmental conditions for optimal Feulgen hydrolysis. In: Weid GL, Bahr GF, eds. Introduction to quantitative cytochemistry II. New York: Academic Press, 1970.

- Allison DC, Ridolpho PF, Rasch EM, Rasch RW, Johnson TS. Increased accuracy of absorption cytophotometric values by control of stain intensity. $J$ Histochem Cytochem 1981;29:1219-28.

7 Goldstein DJ. Aspects of scanning microdensitometry I. Stray light (glare). J Microsc 1970;92:1-16.

${ }^{8}$ Bedi KS, Goldstein DJ. Microdensitometric and autoradiographic comparison of the DNA contents of foetal and adult rat liver nuclei. Histochemistry 1978;55:63-74.

' Cornelisse CJ, de Koning HR, Moolenaar AJ, van de Velde CJ, Ploem JS. Image and flow cytometric analysis of DNA content in breast cancer. Relation to estrogen receptor content and lymph node involvement. Anal Quant Cytol 1984;6:9-19.

${ }^{10}$ Lane N, Fenoglio CM, Kaye GI, Pascal RR. Defining the precursor tissue of ordinary large bowel carcinoma: Implications for cancer prevention. In: Lipkin M, Good RA, eds. Gastrointestinal tract cancer New York: Plenum Medical Book Co, 1978:295-324.

"Kalus M. Carcinoma and adenomatous polyps of the colon and rectum in biopsy and organ tissue culture. Cancer 1972;30:972-82.

Requests for reprints to: Professor R Whitehead, Department of Pathology, Flinders Medical Centre, Bedford Park 5042, South Australia. 